\title{
Real-World Effectiveness of Disease-Modifying Therapies in Korean Patients with Relapsing Multiple Sclerosis
}

\author{
Su-Hyun Kim ${ }^{\mathrm{a}}$ \\ Min Su Park \\ Woojun Kim ${ }^{c}$ \\ So-Young Huh ${ }^{\mathrm{d}}$ \\ Hyun-June Shin ${ }^{\text {a* }}$ \\ Jae-Won Hyun ${ }^{\mathrm{a}}$ \\ Ho Jin Kim ${ }^{a}$ \\ ${ }^{a}$ Department of Neurology, \\ Research Institute and Hospital \\ of National Cancer Center, \\ Goyang, Korea \\ bDepartment of Neurology, \\ College of Medicine, \\ Yeungnam University, \\ Gyeongsan, Korea \\ 'Department of Neurology, \\ The Catholic University \\ of Korea College of Medicine, \\ Seoul, Korea \\ dDepartment of Neurology, \\ Kosin University College of Medicine, \\ Busan, Korea
}

Background and Purpose This study assessed the long-term outcomes of disease-modifying therapies (DMTs) in Korean multiple sclerosis (MS) patients treated in real-world clinical settings in Korea.

Methods We retrospectively evaluated the medical records of 160 patients with an initial diagnosis of clinically isolated syndrome or relapsing-remitting MS who were treated for at least 2 years. A status of 3 for no evidence of disease activity (NEDA3) was defined as no relapse, disability progression, or active lesions in annual magnetic resonance imaging (MRI) evaluations.

Results Patients who were initially treated with interferon $\beta(n=152)$, glatiramer acetate $(n=6)$, or teriflunomide $(n=2)$ were included. The mean disease duration was 8.2 years. Compared to pretreatment, annualized relapse rates were significantly reduced after treatment [from $1.0 \pm 0.8$ to $0.2 \pm 0.4$ (mean \pm standard deviation), $p<0.001$ ]. At the follow-up, 79 patients (49\%) had changed their treatment regimen due to lack of efficacy (33\%), side effects (14\%), or other reasons (2\%). Disability progression was observed in $18 \%$ of the patients over a mean treatment duration of 5.7 years. After 2 years, NEDA3 was observed in $38 \%$ of the patients. Loss of NEDA3 at 2 years was associated with long-term disability progression [odds ratio $(\mathrm{OR})=17.975, p=0.003]$. Poor response to first-line treatment was independently associated with a delay in treatment from disease onset $(\mathrm{OR}=1.238, p=0.049)$ and 10 or more brain lesions in the initial MRI $(\mathrm{OR}=3.648, p=0.047)$.

Conclusions This study has provided real-world evidence that DMTs are effective in reducing disease activity and disability progression in Korean MS patients.

Key Words disease-modifying therapy, multiple sclerosis, Korea, treatment outcomes.
Received April 24, 2018

Revised July 13, 2018

Accepted July 13, 2018

\section{Correspondence}

Ho Jin Kim, MD, PhD

Department of Neurology,

Research Institute and

Hospital of National Cancer Center,

323 Ilsan-ro, Ilsandong-gu,

Goyang 10408, Korea

Tel $+82-31-920-2438$

Fax +82-31-925-5524

E-mail hojinkim@ncc.re.kr

*Current affiliation: Department of

Neurology, Chonbuk National University

Medical School, Jeonju, Korea

\section{INTRODUCTION}

There have recently been substantial advances in the treatment of relapsing-remitting multiple sclerosis (RRMS). Numerous disease-modifying therapies (DMTs) are currently available for early aggressive interventions in patients with RRMS. Although general conclusions have been drawn about the efficacy of DMTs from randomized controlled trials, observational studies in real-world settings are essential for documenting the long-term impact of DMT in the wider population. Such studies can provide insight into the predictors of a response to treatment for MS, which will be useful for daily treatment decision-making. Numerous observational studies of MS patients receiving DMTs in Western countries have shown long-term treatment benefits and identified several predictors of a poor prognosis. ${ }^{1-5}$ The prevalence of MS is lower in Asia than in Western countries, and there is less data available about the long-term treatment outcomes of DMTs and predictors of a treatment response in Asian MS patients.

MS is considered to be a rare disease in Korea. ${ }^{6}$ All Korean MS patients are eligible to

(a) This is an Open Access article distributed under the terms of the Creative Commons Attribution Non-Commercial License (https://creativecommons.org/licenses/by-nc/4.0) which permits unrestricted non-commercial use, distribution, and reproduction in any medium, provided the original work is properly cited. 
receive financial support for medical care from the government. The treatment era for RRMS began in 1993, and 11 DMTs have been approved by the Korean Food and Drug Administration in line with the global expansion of therapeutic options for MS. However, the effect of these drugs in Korean patients, the remission of disease activity, and the long-term benefits with respect to disabilities are not fully understood. The present study therefore aimed to determine the long-term impact of DMTs on the remission of disease activity and the prevention of disability progression in Korean MS patients. We also estimated 1) how many patients achieve a status of no evidence of disease activity (NEDA), as measured by relapses, disability, and magnetic resonance imaging (MRI) over 2 years, 2) whether the loss of NEDA within 2 years can predict long-term disability progression, 3) how many patients require treatment escalation following first-line treatment, and 4) predictors of a poor response to first-line treatment.

\section{METHODS}

\section{Patients and definitions}

This was a multicenter retrospective cohort study conducted in four MS referral hospitals in Korea. We included consecutive patients, seen up until July 2017, who had an initial diagnosis of clinically isolated syndrome (CIS) or RRMS according to the $2005 \mathrm{McDonald}_{\text {criteria }}{ }^{7}$ and were treated for a minimum of 2 years. CIS was defined as an initial clinical demyelinating event with findings in brain MRI consistent with MS. ${ }^{8}$ We excluded patients with progressive-onset disease without relapses and patients for whom treatment with mitoxantrone was initiated. We performed a retrospective analysis of the demographic information of the enrolled patients, key episodes in their MS course (relapses, onset of the progressive course, and time point when the disability was assigned as being irreversible), MRI findings (whether there were 10 or more high-intensity lesions in initial T2-weighted $\mathrm{MRI}^{4}$ and new/enlarging T2-weighted lesions or gadolinium-enhanced lesions in follow-up MRI), and treatment history. The number of lesions in MRI and the disability status [based on the Expanded Disability Status Scale (EDSS)] were evaluated by neurologists at each center. The MS severity score (MSSS), calculated by adjusting the EDSS score according to disease duration, was used as a marker of disease severity. The MSSS reflects disease severity, with higher scores reflecting a shorter time to reach a particular level of disability, and allows comparisons between multiple patient cohorts. ${ }^{10}$ The present study was approved by the ethics committees of the participating hospitals (IRB No. NCC2014-0146, KC17TEDI0427, 201607040).
The first MRI scans were obtained within 3 months of the onset of CIS. Brain MRI was performed every 1-3 years after the initial MRI and subsequent relapses. Ninety-seven patients underwent brain MRI annually and were thus assigned an NEDA status of 3 (NEDA3; corresponding to no relapses, disability progression, or new/enlarging MRI lesions) for the initial 2 years, as opposed to a status of 3 for evidence of disease activity (EDA3) when at least one of these conditions was present. The MRI studies were conducted at each center using a 1.5-tesla or 3.0-tesla scanner, and the slice thickness varied from 3 to $5 \mathrm{~mm}$. MRI scans consisted of at least T1- and T2-weighted images, FLAIR images, and T1-weighted images with gadolinium enhancement.

An MS relapse was defined as the occurrence, recurrence, or worsening of symptoms of neurological dysfunction lasting for more than $24 \mathrm{~h}$; this usually progressed to partial or complete remission. ${ }^{11}$ A poor response to first-line treatment was defined as 3 months of confirmed progression of the EDSS score and/or switching of therapy due to ongoing disease activity (i.e., clinical relapse or MRI activity). Disability progression was defined according to the baseline EDSS score. To reduce the inherent noise in transitions between EDSS scores of 0 and 1.0, increases in the EDSS score of at least 1.5, 1.0, and 0.5 points were required for subjects with baseline EDSS scores of $0,1.0-5.0$, and $>5.0$, respectively. ${ }^{12}$ Disability progression at the last follow-up was confirmed when the patient next visited, in accordance with the routine clinical practice (once every 3 months) in the absence of relapse at the time of assessment.

\section{Statistical analysis}

Descriptive statistics (e.g., mean \pm standard-deviation values) were used for continuous variables. Categorical variables were described as numbers and percentages of subjects in each group. Logistic regression was used to determine whether baseline demographic, clinical, radiological, and laboratory features of the patients were correlated with EDA3 over the initial 2 years, disability progression, or poor response to first-line treatment. Univariable analyses were conducted separately for potentially related factors. Variables with $p$ values of $<0.2$ in the univariable analyses were included in the multivariable model. The final model was developed using a backward variable selection method with an elimination criterion of 0.05. STATA SE software (version 10.0, StataCorp., College Station, TX, USA) was used for all analyses. Two-sided $p$ values of $<0.05$ were considered statistically significant. 


\section{RESULTS}

This study enrolled 160 patients: 3 with CIS, 152 with RRMS, and 5 with secondary progressive multiple sclerosis (SPMS). At the initiation of DMT, 13 had CIS and 147 had RRMS. The baseline characteristics of subjects are summarized in Table 1. Their age at the onset of MS was 29.2 29.7 years, the femaleto-male ratio was 2.07 , and the disease duration from clinical onset to the last visit was $8.2 \pm 5.2$ years. Treatment was started at a median of 1 year (range, 0-22 years) after the clinical onset of MS, and the treatment duration was $5.7 \pm 3.3$

Table 1. Clinical and demographic characteristics of the study patients with MS $(n=160)$

\begin{tabular}{|c|c|}
\hline Characteristic & Value \\
\hline Age at onset, years & $29.2 \pm 9.7$ \\
\hline Age at initiation of treatment, years & $31.1 \pm 9.8$ \\
\hline Female & $108(68)$ \\
\hline Disease duration, years & 8 [2-29] \\
\hline $\begin{array}{l}\text { Time from disease onset to treatment initiation, } \\
\text { years }\end{array}$ & $1[0-22]$ \\
\hline Treatment duration, years & $5[2-18]$ \\
\hline \multicolumn{2}{|l|}{ Clinical phenotype at initiation of DMT } \\
\hline $\mathrm{CIS}$ suggestive of MS & $13(29)$ \\
\hline RRMS & $147(71)$ \\
\hline \multicolumn{2}{|l|}{ Clinical phenotype at last clinical visit } \\
\hline CIS suggestive of MS & $3(23)$ \\
\hline RRMS & $152(74)$ \\
\hline Secondary progressive MS & $5(3)$ \\
\hline ARR before therapy & 0.84 [0.09-3] \\
\hline ARR after therapy & $0[0-2.5]$ \\
\hline Presence of drug switching & $79(49)$ \\
\hline Lack of effectiveness & $53(33)$ \\
\hline Adverse effects & $23(14)$ \\
\hline Others & $3(2)$ \\
\hline EDSS score at initiation of treatment & $2.0[0-5.0]$ \\
\hline EDSS score at last follow-up & $1.5[0-7.5]$ \\
\hline $\begin{array}{l}\text { Patients with worsening of EDSS score after } \\
\text { treatment }\end{array}$ & $29(18)$ \\
\hline MSSS at initiation of treatment & $4.27[0.24-8.64]$ \\
\hline MSSS at last follow-up & $1.77[0.04-9.08]$ \\
\hline Patients NEDA3 at 2 years* & 37/97 (38) \\
\hline Patients with CSF oligoclonal band* & $42 / 84(50)$ \\
\hline Patients with 10 brain lesions in initial MRI* & $66 / 129(51)$ \\
\hline
\end{tabular}

Data are mean \pm standard-deviation, $n(\%)$, or median [range] values. *For parameters that were not evaluated in all 160 patients, the number of patients for whom these data were available is indicated. ARR: annualized relapse rate, CIS: clinically isolated syndrome, CSF: cerebrospinal fluid, DMT: disease-modifying therapy, EDSS: Extended Disability Status Scale, MRI: magnetic resonance imaging, MS: multiple sclerosis, MSSS: multiple sclerosis severity score, NEDA3: status of 3 for no evidence of disease activity, RRMS: relapsing-remitting multiple sclerosis. years. Of 47 patients for whom treatment was initiated at the first attack, 12 (26\%) had progressed to clinically definite MS. Compared to the pretreatment phase, the annualized relapse rate $(A R R)$ was significantly reduced during the treatment (from $1.0 \pm 0.8$ to $0.2 \pm 0.4, p<0.001$ ). The median MSSS at the time of the initiation of DMTs was 4.27 (range, $0.24-8.64$ ) in 89 patients for whom at least 1 year had elapsed between disease onset and treatment, with a median MSSS of 1.77 (range, 0.04-9.08) at the last follow-up. Globally, significant worsening in EDSS score during the treatment period was observed in 29 patients (18\%). Of the 106 and 35 patients who were treated for at least 5 and 10 years, respectively, 26 patients (25\%) and 14 patients (40\%) experienced disability progression. At the last follow-up, 30 patients (19\%) had an EDSS score of $\geq 3$ and 9 patients (6\%) had an EDSS score of $\geq 6.0$. Among 60 patients with a disease duration of at least 10 years, 5 (8\%) progressed to SPMS and 8 (13\%) had an EDSS score of $\geq 6$.

Most of the patients were initially treated with interferon $\beta$ (IFN $\beta$ ) ( $n=152$ ) [91 with subcutaneous (s.c.) IFN $\beta-1 b, 51$ with s.c. IFN $\beta-1 \mathrm{a}$, and 10 with intramuscular (i.m.) IFN $\beta$ 1a), while 6 were started on glatiramer acetate, and 2 on teriflunomide (Fig. 1A). During the follow-up, 81 patients (51\%) had remained on their first treatment regimen, while 79 patients (49\%) had changed their treatment regimen due to lack of efficacy $(n=53,67 \%)$, side effects $(n=23,29 \%)$, or other reasons $(n=3,4 \%)$. Thirty-four $(21 \%)$ patients escalated to a second-line DMT [natalizumab $(n=4)$, alemtuzumab $(n=$ 17 ), fingolimod $(n=8)$, or mitoxantrone $(n=7)]$. In patients switching DMTs due to lack of efficacy, the median time to the first switch (including lateral switching or escalation) was 2 years (range, 3-17 years), and the median time to the second switch was 1.4 years (range, 5-8 years). The first drug switch due to a lack of efficacy was observed after a median of 2 attacks (range, 0-25 attacks) during first-line treatment.

Four patients switched to mitoxantrone due to the progression of disability without clinical relapse when receiving the first-line injectable treatment. Since alemtuzumab and natalizumab were launched in Korea in 2015, the median time from the initiation of the first-line drug to these higher potency drugs was 4 years (range, 1.3-17 years), and the median treatment duration was 15 months. Fingolimod was not eligible for reimbursement until 2017, but 8 patients started this treatment in 2013 via the Drug-Free Communities Support Program. Among them, one switched to natalizumab due to persistent lymphopenia and one switched to alemtuzumab due to lack of efficacy. Of 29 patients who switched to fingolimod, natalizumab, or alemtuzumab, ongoing disease activity (i.e., clinical relapse or MRI activity) was observed in 10 patients (34\%), and significant disability worsening was found in only 


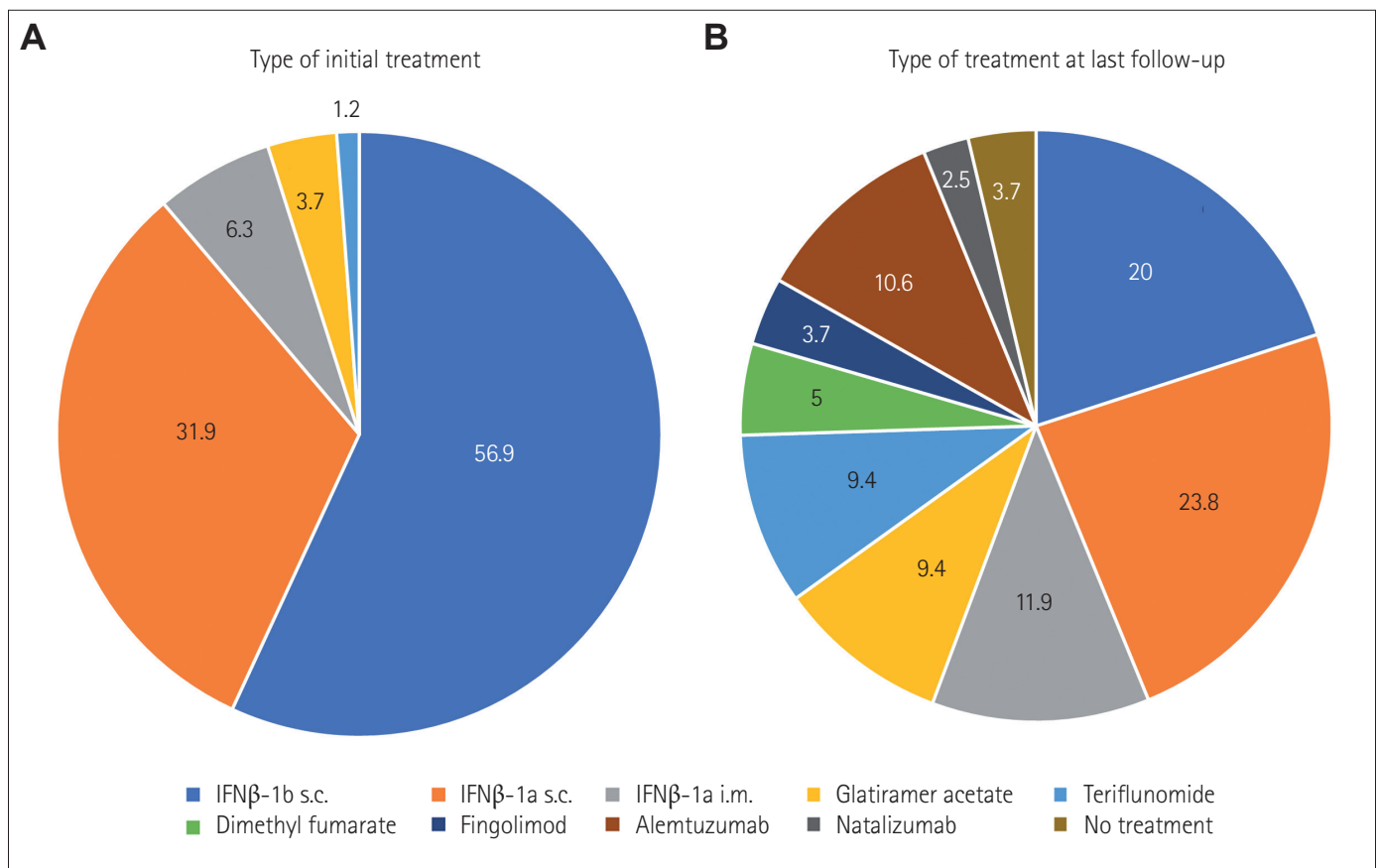

Fig. 1. Type of treatment at the initiation of treatment (A) and at last the follow-up (B). IFN $\beta$ : interferon $\beta$, i.m.: intramuscular, s.c.: subcutaneous.

1 patient (3\%) during a median of 18 months of treatment with these higher potency drugs. All 4 patients treated with natalizumab achieved an NEDA3 during a median of 18 months (range, 18-20 months), and no progressive multifocal leukoencephalopathy occurred. Of the 23 patients who switched drugs due to side effects such as flu-like symptoms, injectionsite reactions, or laboratory abnormalities, 11 switched from s.c. IFN $\beta$-1b or IFN $\beta$ - 1 a to i.m. IFN $\beta$ - 1 a, 8 switched from injectable drugs to oral first-line agents, and 4 switched from s.c. IFN $\beta-1 b$ or IFN $\beta$-1a to glatiramer acetate. The treatment at the last follow-up consisted of first-line injectable therapies $(n=104)$, first-line oral therapies $(n=23)$, second-line oral therapies $(n=6)$, second-line injectable therapies $(n=21)$, or no therapy ( $n=6)$ (Fig. 1B). At the follow-up there were no reports of serious adverse clinical events leading to treatment discontinuation.

In our cohort, 97 patients were eligible for an NEDA3 evaluation during the initial 2 years, and this resulted in NEDA3 being found in 37 of the patients (38\%). To evaluate the utility of NEDA3 at 2 years as a predictor of long-term disease progression, we conducted subgroup analysis in patients who were treated for at least 5 years $(n=106)$. The sensitivity, specificity, positive predictive value (PPV), and negative predictive value of EDA3 at 2 years for disability progression over a median of 7 years of treatment were $96 \%, 48 \%, 38 \%$, and $97 \%$, respectively. In univariable analyses, disease duration [odds ratio $(\mathrm{OR})=1.194,95 \%$ confidence interval $(\mathrm{CI})=1.080$ $1.320, p=0.001]$ and EDA 3 at 2 years were associated with progression of the EDSS score over a median of 7 years of treat- ment $(\mathrm{OR}=20.167,95 \% \mathrm{CI}=2.573-158.058, p=0.004)$ (Table 2). In multivariable analyses, $\mathrm{EDA} 3$ at 2 years $(\mathrm{OR}=14.444$, $95 \% \mathrm{CI}=1.770-117.819, p=0.013)$ was independently associated with disability progression.

Our cohort included 54 patients (34\%) classified as poor responders to first-line treatment. Multivariable logistic regression analyses revealed that poor responses were independently associated with time (per year longer) from the disease onset to treatment $(\mathrm{OR}=1.238,95 \% \mathrm{CI}=1.001-1.533$, $p=$ 0.049 ) and the presence of 10 or more brain lesions in the initial MRI (OR=3.648, 95\% CI $=1.018-13.071, p=0.047)$ (Table 3).

\section{DISCUSSION}

The main aim of this study was to determine the long-term outcomes of DMTs in a cohort of Korean patients with MS and the predictors of a poor response to first-line treatment. Most of the patients (82\%) achieved long-term stabilization of their disability (based on the EDSS scores) during 6 years of treatment. DMTs reduced the ARR by $80 \%$. Escalation to higher potency therapy was observed in $21 \%$ of the patients, which stabilized the disease activity in most of them. Among 158 patients who started first-line injectable treatments, 23 patients (15\%) switched their drug treatment regimens to lowfrequency administration, other types of injectable drugs, or oral agents due to their simplicity and convenience.

Our previous report on the clinical characteristics of 105 Korean MS patients demonstrated that the clinical pattern of 
Table 2. Results from univariate and multivariate logistic regression models of patients with disability progression after receiving at least 5 years of treatment $(n=106)$

\begin{tabular}{|c|c|c|c|c|}
\hline \multirow{2}{*}{ Variable } & \multicolumn{2}{|c|}{ Univariate analysis } & \multicolumn{2}{|c|}{ Multivariate analysis } \\
\hline & OR $(95 \% \mathrm{Cl})$ & $p$ & OR $(95 \% \mathrm{Cl})$ & $p$ \\
\hline Age at disease onset & $0.965(0.919-1.013)$ & 0.154 & & \\
\hline Age at initiation of DMT & $0.991(0.947-1.037)$ & 0.695 & & \\
\hline Sex, female & $0.755(0.308-1.853)$ & 0.540 & & \\
\hline Initiation of DMT after second attack, yes & $4.237(0.920-19.509)$ & 0.064 & & \\
\hline Disease duration, per year longer & $1.194(1.080-1.320)$ & $0.001^{*}$ & & \\
\hline ARR before DMT & $1.167(0.476-2.862)$ & 0.386 & & \\
\hline \multicolumn{5}{|l|}{ Attack at onset } \\
\hline Brainstem attack, yes vs. no & $0.670(0.224-2.005)$ & 0.474 & & \\
\hline Optic neuritis, yes vs. no & $1.094(0.380-3.153)$ & 0.868 & & \\
\hline Myelitis, yes vs. no & $0.965(0.371-2.508)$ & 0.965 & & \\
\hline EDA3 over 2 years, yes & $20.167(2.573-158.058)$ & $0.004^{*}$ & $14.444(1.770-117.879)$ & $0.013^{*}$ \\
\hline$\geq 10$ high-intensity T2-weighted lesions in initial MRI, yes & $1.949(0.605-6.280)$ & 0.264 & & \\
\hline CSF oligoclonal band, positive & $1.614(0.476-5.470)$ & 0.442 & & \\
\hline
\end{tabular}

Multivariate analysis restricted to 88 patients with complete data for all variables ( $p<0.2$ in the univariate analysis).

*Significant findings.

ARR: annualized relapse rate, Cl: confidence interval, CSF: cerebrospinal fluid, DMT: disease-modifying therapy, EDA3: status of 3 for evidence of disease activity, OR: odds ratio.

Table 3. Results from univariable and multivariable logistic regression models of patients with poor responses to first-line therapy

\begin{tabular}{|c|c|c|c|c|}
\hline \multirow{2}{*}{ Variable } & \multicolumn{2}{|c|}{ Univariable analysis } & \multicolumn{2}{|c|}{ Multivariable analysis } \\
\hline & OR $(95 \% \mathrm{Cl})$ & $p$ & OR $(95 \% \mathrm{Cl})$ & $p$ \\
\hline Age at disease onset & $0.972(0.938-1.031)$ & 0.120 & & \\
\hline Age at initiation of DMT & $0.972(0.938-1.007)$ & 0.120 & & \\
\hline Sex, female & $0.574(0.289-1.142)$ & 0.114 & $0.266(0.071-1.0)$ & 0.05 \\
\hline Time from disease onset to initiation of treatment & $1.158(1.050-1.277)$ & $0.003^{*}$ & $1.238(1.001-1.533)$ & $0.049^{*}$ \\
\hline ARR before DMT & $1.167(0.476-2.862)$ & 0.386 & & \\
\hline \multicolumn{5}{|l|}{ Attack at onset } \\
\hline Brainstem attack, yes vs. no & $0.974(0.469-2.022)$ & 0.944 & & \\
\hline Optic neuritis, yes vs. no & $0.695(0.316-1.531)$ & 0.367 & & \\
\hline Myelitis, yes vs. no & $1.125(0.5338-2.355)$ & 0.754 & & \\
\hline$\geq 10$ high-intensity $\mathrm{T} 2$-weighted lesions in initial MRI, yes & $2.273(1.014-5.097)$ & $0.046^{*}$ & $3.648(1.018-13.071)$ & $0.047^{*}$ \\
\hline CSF oligoclonal band, positive & $2.750(1.056-7.164)$ & $0.038^{*}$ & & \\
\hline
\end{tabular}

Multivariable analysis restricted to the 67 patients with complete data for all variables ( $p<0.2$ in the univariate analysis).

*Significant findings.

ARR: annualized relapse rate, $\mathrm{Cl}$ : confidence interval, CSF: cerebrospinal fluid, DMT: disease-modifying therapy, OR: odds ratio.

MS in Korea did not differ fundamentally from that seen in Western countries. ${ }^{13}$ Before 2015, mitoxantrone was the only second-line treatment for which reimbursement was provided in Korea. This meant that our previous study could only evaluate the efficacy of IFN $\beta$ treatment, and 14 of 88 patients (16\%) showed disability progression during approximately 3 years. Alemtuzumab and natalizumab were launched in Korea in 2015, while fingolimod was approved for reimbursement from the government in 2017. Eight of the patients in the present cohort started fingolimod in 2013 via the DrugFree Communities Support Program. Although the treatment duration was longer in the present study (mean, 6 years) than in our previous study, the proportion of patients with disability progression was similar (18\% of 160 patients), which may be attributed to the effect of escalation with these therapies. Of the patients with a disease duration longer than 10 years $(n=60)$, the proportions of patients who progressed to SPMS (8\%) and reached an EDSS score of $6(13 \%)$ were lower than those found in Western patients for the natural history of MS, with approximately $60 \%$ of the patients who were followed for $11-25$ years from onset showing these outcomes. ${ }^{14}$

It could be argued that the more-favorable outcome in the present cohort is due to the effect of racial differences in MS disease activity, and is only partially explained by treatment 
effects. Piccolo et al. ${ }^{15}$ reported that the median MSSS was significantly lower in Japanese patients (3.34) than in patients from the UK (5.87). MS may indeed be associated with less disability in Japan than in the UK, but because of differences in the number of patients (104 in Japan vs. 1,148 in the UK), the proportion of primary progressive MS patients (3\% in Japan vs. $12.9 \%$ in the UK), and the proportion of patients receiving DMTs ( $83.7 \%$ in Japan vs. 17\% in the UK) between the two cohorts, it is difficult to conclude that the MS disease activity is milder in Japanese patients than in UK patients.

In the present study, the median MSSS was 4.27 at the time of initiating DMTs, which is comparable to that of global cohorts, although the EDSS scores at the initiation of treatment may be confounded by the incomplete recovery from a prior attack in some patients. At the last follow-up, based on EDSS scores that were confirmed after $\geq 3$ months, we observed a significantly lower median MSSS (of 1.77) than that at the time of DMT initiation. These findings suggest that the clinical course of our patients had been changed by the effectiveness of DMTs. The early initiation of treatment in our cohort (median, 1 year) may have stabilized the disease course and resulted in lower MSSSs during the ensuing years.

The subgroup analysis revealed NEDA3 at 2 years in 38\% of our patients, which is slightly higher than the reported percentages (17.9-27.5\%) of NEDA3 over 2 years in patients with RRMS in Western countries. ${ }^{2,16}$ The inclusion of CIS patients (8\%) in the present study may have contributed to these higher rates, but this factor alone cannot explain the higher rates of NEDA3 observations in the present study. The retrospective design of our study meant that serial MRI scans were not obtained using the same MRI system and imaging protocols. In addition, a section thickness of $5 \mathrm{~mm}$ was used in the MRI protocols for some patients, and this is greater than that of $\leq 3 \mathrm{~mm}$ used in the standard protocol. These limitations of the MRI assessments might have resulted in low sensitivity for monitoring disease activity based on MRI findings and consequently contributed to the high rate of NEDA3 at 2 years that was observed in our study.

NEDA3 has become an important treatment goal and outcome measure in clinical trials, ${ }^{17}$ but its utility as a predictor of long-term disease progression remains unclear. In the present study, EDA3 over 2 years had a negative impact on the risk of disability progression during a median of 7 years of treatment. However, the PPV of EDA3 over 2 years was only $38 \%$, and the presence of EDA3 over 2 years did not necessarily predict future disability progression at the individual level. This finding is in agreement with recent studies in Western countries finding that EDA3 over 1-2 years had good or moderate sensitivity but relatively low specificity for the prediction of long-term disability progression..$^{16,18}$ Regarding the low specificity of EDA3, Río et al. ${ }^{19}$ recently reported that minimal disease activity [i.e., one relapse without an impact on disability, or the presence of minimal isolated radiological activity (one or two new T2-weighted lesions, or a single gadolinium-enhanced lesion)] did not confer a significant risk of the EDSS score worsening over a mean of 6.7 years of follow-up. Furthermore, the disease activity before the drug becomes effective should not be considered as EDA3 on treatment. ${ }^{20}$ Finally, even if there is significant disease activity at the beginning of treatment, switching to more efficacious therapies within an appropriate timeframe can prevent the further deterioration of disability. ${ }^{21}$

In our cohort, $34 \%$ of the patients were classified as poor responders (switching drugs due to lack of efficacy or disability progression) to first-line treatment. This poor response to first-line treatment was independently associated with delayed initiation of DMTs (per year longer) $(\mathrm{OR}=1.238$, $p=$ 0.049 ), which is consistent with current treatment guidelines to start DMTs early in order to obtain more-favorable outcomes. The presence of at least 10 high-intensity T2-weighted brain lesions in the initial MRI $(\mathrm{OR}=3.348, p=0.047)$ also increased the risk of a poor response to first-line treatment. A large observational study in Spain found that the presence of at least 10 high-intensity T2-weighted brain MRI lesions at baseline is the most-robust predictive factor for the conversion to MS and disability accumulation after controlling for all of the other variables examined. ${ }^{4}$ Therefore, the presence of a large number of brain lesions at baseline may indicate high disease activity, and so these patients may require a high level of vigilance for the drug response during treatment and should be considered for early escalation if required.

The limitations of this study include its retrospective design and small sample. The small proportion of patients with SPMS or with an EDSS score of $\geq 6$ in our cohort may have been due to selection bias. Since we included patients who had been treated for at least 2 years, patients who already had SPMS during the initiation of DMTs and did not maintain the drug for more than 2 years were likely to have been excluded from this study. Meanwhile, because the four included centers are referral centers in Korea, unintentional selection bias may have caused a greater inclusion of patients with on-going disease activity despite first-line treatment. In addition, because the use of second-line treatment with reimbursement has only been available in Korea since 2015, the duration of treatment of the higher-potency drugs may be insufficient for evaluating efficacy and safety. Nevertheless, this study is the first to provide insight into the real-world longterm benefit of DMTs in Korean patients with MS, and the proportion of patients with NEDA3 at 2 years based on an an- 
nual MRI evaluation and its prognostic value for long-term disability.

In conclusion, the real-world benefits of DMTs observed in Korean patients seem to be similar to those found in previous observational studies and clinical trials in Western countries. ${ }^{3,22-24}$ Starting DMTs early could therefore lead to morefavorable outcomes. Additionally, patients with a large number of brain lesions at baseline may need to be monitored more carefully to track the effects of first-line treatments. Future studies should involve longer follow-ups, have larger samples, and examine newly developed second-line treatments in greater depth.

\section{Conflicts of Interest}

Park MS, Kim W, Huh SY, Shin HJ, and Hyun JW report no conflicts of interest. Kim SH has received a grant from the National Research Foundation of Korea. Kim HJ has given lectures at, provided consultation to, and received honoraria from Bayer Schering Pharma, Biogen, Celltrion, Eisai, Genzyme, HanAll BioPharma, MedImmune, Merck Serono, Novartis, Teva-Handok, and UCB; received a grant from the Ministry of Science \& ICT; accepted research funding from Genzyme, Kael-GemVax, Merck Serono, Teva-Handok and UCB; serves on a steering committee for MedImmune; is a coeditor for the Multiple Sclerosis Journal-Experimental, Translational and Clinical; and is an associated editor for the Journal of Clinical Neurology.

\section{Acknowledgements}

This work was supported by the National Research Foundation of Korea (Grant No. NRF-2016R1D1A1A09916480).

\section{REFERENCES}

1. Kappos L, Kuhle J, Multanen J, Kremenchutzky M, Verdun di Cantogno E, Cornelisse P, et al. Factors influencing long-term outcomes in relapsing-remitting multiple sclerosis: PRISMS-15. J Neurol Neurosurg Psychiatry 2015;86:1202-1207.

2. University of California, San Francisco MS-EPIC Team, Cree BA, Gourraud PA, Oksenberg JR, Bevan C, et al. Long-term evolution of multiple sclerosis disability in the treatment era. Ann Neurol 2016;80: 499-510.

3. Jokubaitis VG, Spelman T, Kalincik T, Lorscheider J, Havrdova E, Horakova $\mathrm{D}$, et al. Predictors of long-term disability accrual in relapse-onset multiple sclerosis. Ann Neurol 2016;80:89-100.

4. Tintore M, Rovira À, Río J, Otero-Romero S, Arrambide G, Tur C, et al. Defining high, medium and low impact prognostic factors for developing multiple sclerosis. Brain 2015;138:1863-1874.

5. Trojano M, Pellegrini F, Paolicelli D, Fuiani A, Zimatore GB, Tortorella $\mathrm{C}$, et al. Real-life impact of early interferon beta therapy in relapsing multiple sclerosis. Ann Neurol 2009;66:513-520.

6. Kim NH, Kim HJ, Cheong HK, Kim BJ, Lee KH, Kim EH, et al. Prevalence of multiple sclerosis in Korea. Neurology 2010;75:1432-1438.

7. Polman CH, Reingold SC, Edan G, Filippi M, Hartung HP, Kappos L, et al. Diagnostic criteria for multiple sclerosis: 2005 revisions to the "McDonald Criteria". Ann Neurol 2005;58:840-846.
8. O’Riordan JI, Thompson AJ, Kingsley DP, MacManus DG, Kendall BE, Rudge $\mathrm{P}$, et al. The prognostic value of brain MRI in clinically isolated syndromes of the CNS. A 10-year follow-up. Brain 1998;121:495-503.

9. Roxburgh RH, Seaman SR, Masterman T, Hensiek AE, Sawcer SJ, Vukusic S, et al. Multiple Sclerosis Severity Score: using disability and disease duration to rate disease severity. Neurology 2005;64:1144-1151.

10. Pachner AR, Steiner I. The multiple sclerosis severity score (MSSS) predicts disease severity over time. J Neurol Sci 2009;278:66-70.

11. Confavreux C, Vukusic S, Adeleine P. Early clinical predictors and progression of irreversible disability in multiple sclerosis: an amnesic process. Brain 2003;126:770-782.

12. Calabresi PA, Radue EW, Goodin D, Jeffery D, Rammohan KW, Reder AT, et al. Safety and efficacy of fingolimod in patients with relapsingremitting multiple sclerosis (FREEDOMS II): a double-blind, randomised, placebo-controlled, phase 3 trial. Lancet Neurol 2014;13:545556.

13. Kim SH, Huh SY, Kim W, Park MS, Ahn SW, Cho JY, et al. Clinical characteristics and outcome of multiple sclerosis in Korea: does multiple sclerosis in Korea really differ from that in the Caucasian populations? Mult Scler 2013;19:1493-1498.

14. Weinshenker BG, Bass B, Rice GP, Noseworthy J, Carriere W, Baskerville J, et al. The natural history of multiple sclerosis: a geographically based study. I. Clinical course and disability. Brain 1989;112:133-146.

15. Piccolo L, Kumar G, Nakashima I, Misu T, Kong Y, Wakerley B, et al. Multiple sclerosis in Japan appears to be a milder disease compared to the UK. J Neurol 2015;262:831-836.

16. Rotstein DL, Healy BC, Malik MT, Chitnis T, Weiner HL. Evaluation of no evidence of disease activity in a 7-year longitudinal multiple sclerosis cohort. JAMA Neurol 2015;72:152-158.

17. Nixon R, Bergvall N, Tomic D, Sfikas N, Cutter G, Giovannoni G. No evidence of disease activity: indirect comparisons of oral therapies for the treatment of relapsing-remitting multiple sclerosis. Adv Ther 2014;31:1134-1154.

18. Uher T, Havrdova E, Sobisek L, Krasensky J, Vaneckova M, Seidl Z, et al. Is no evidence of disease activity an achievable goal in MS patients on intramuscular interferon beta-1a treatment over long-term followup? Mult Scler 2017;23:242-252.

19. Río J, Rovira À, Tintoré M, Otero-Romero S, Comabella M, Vidal-Jordana Á, et al. Disability progression markers over 6-12 years in interferon- $\beta$-treated multiple sclerosis patients. Mult Scler 2018;24:322330.

20. Erbayat Altay E, Fisher E, Jones SE, Hara-Cleaver C, Lee JC, Rudick RA. Reliability of classifying multiple sclerosis disease activity using magnetic resonance imaging in a multiple sclerosis clinic. JAMA Neurol 2013;70:338-344.

21. Ransohoff RM, Hafler DA, Lucchinetti CF. Multiple sclerosis-a quiet revolution. Nat Rev Neurol 2015;11:134-142.

22. Hupperts R, Ghazi-Visser L, Martins Silva A, Arvanitis M, Kuusisto $\mathrm{H}$, Marhardt K, et al. The STAR study: a real-world, international, observational study of the safety and tolerability of, and adherence to, serum-free subcutaneous interferon $\beta$-1a in patients with relapsing multiple sclerosis. Clin Ther 2014;36:1946-1957.

23. Carroll WM. Clinical trials of multiple sclerosis therapies: improvements to demonstrate long-term patient benefit. Mult Scler 2009;15: 951-958.

24. Freedman MS. Long-term follow-up of clinical trials of multiple sclerosis therapies. Neurology 2011;76:S26-S34. 